\title{
Symmetry Breaking at Magnetic Surfaces and Interfaces
}

\author{
Z.Q. Qiu \\ Department of Physics \\ University of California at Berkeley, Berkeley, CA 94720 \\ and \\ S.D. Bader \\ Materials Science Division \\ Argonne National Laboratory, Argonne, IL 60439

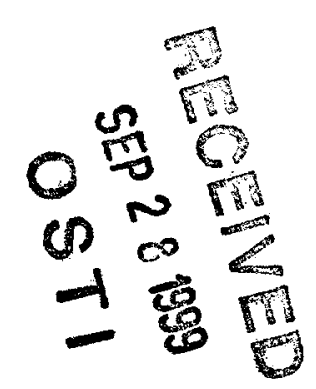

\begin{abstract}
The submitted manuscript has been created by the University of Chicago as Operator of Argonne National Laboratory ("Argonne") under Contract No. W-31-109-ENG-38 with the U.S. Department of Energy. The U.S. Government retains for itself, and others acting on its behalf, a paid-up, nonexclusive, irrevocable worldwide license in said article to reproduce, prepare derivative works, distribute copies to the public, and perform publicly and display publicly, by or on behalf of the Government.
\end{abstract}

Proceedings of the International Symposium on Surface and Interface: Properties of Different Symmetry Crossing, Tokyo, Japan, November 19-21, 1998

Work supported by the U.S. Department of Energy, Basic Energy Sciences-Materials Sciences under contract \#W-31-109-ENG-38 (Argonne) and NSF Contract No. DMR-9805222 (at Berkeley). 


\section{DISCLAIMER}

This report was prepared as an account of work sponsored by an agency of the United States Government. Neither the United States Government nor any agency thereof, nor any of their employees, make any warranty, express or implied, or assumes any legal liability or responsibility for the accuracy, completeness, or usefulness of any information, apparatus, product, or process disclosed, or represents that its use would not infringe privately owned rights. Reference herein to any specific commercial product, process, or service by trade name, trademark, manufacturer, or otherwise does not necessarily constitute or imply its endorsement, recommendation, or favoring by the United States Government or any agency thereof. The views and opinions of authors expressed herein do not necessarily state or reflect those of the United States Government or any agency thereof. 


\section{DISCLAIMER}

Portions of this document may be illegible in electronic image products. Images are produced from the best available original document. 


\title{
Symmetry Breaking at Magnetic Surfaces and Interfaces*
}

\author{
Z. Q. Qiu \\ Department of Physics, University of California at Berkeley, Berkeley, California 94720 \\ and \\ S. D. Bader \\ Materials Science Division, Argonne National Laboratory, Argonne, Illinois 60439
}

Examples are presented of how symmetry breaking enters into consideration of the physical properties of magnetic surfaces and ultrathin films. The role of magnetic anisotropy is discussed to understand: (i) the existence of two-dimensional (2D) magnetic long-ranged order at finite temperature, (ii) magnetization scaling behavior at the Curie transition, (iii) the 2D spin reorientation transition, and (iv) step-induced magnetic behavior. Experimental examples cited include ultrathin magnetic $\mathrm{Fe}$ and $\mathrm{Co}$ overlayer and wedge structures grown onto single crystal subtrates that are either flat or curved to produce vicinal surfaces with a continuous gradient in the step density. Also included is an example of an atomically flat manganite intergrowth that appears as a stacking fault in a bulk single crystal of a naturally layered structure.

Work supported by DOE BES-MS under Contract No. W-31-109-ENG-38 (at Argonne) and DE-AC03-76SF00098 and NSF Contract No. DMR-9805222 (at Berkeley). 


\section{Overview}

The limiting thickness of an ultrathin magnetic film is a two-dimensional (2D) magnetic sheet. In practice such films can be grown epitaxially onto single-crystal substrates, or can appear as inclusions or intergrowths in naturally layered structures. An issue of interest is that in $2 \mathrm{D}$ it is well known theoretically that there is no long-range order (LRO) at finite temperature for the isotropic Heisenberg model. Thus, it is the presence of anisotropy that serves as the symmetry breaking element that permits ferromagnetic order to be observed experimentally. Examples are presented in the present work to highlight the richness of phenomena encountered in quasi-2D magnetic systems. In Sec. II(a) the appearance of ferromagnetic LRO and its scaling properties are considered for epitaxial overlayers of Fe. In Sec. II(b) the 2D spin-reorientation transition is introduced experimentally as a phase transition driven by the change in the dominant magnetic anisotropy with film thickness. In Sec. II(c) the unusual example of ultraflat ferrromagnetic inclusions in naturally layered manganites is considered where finite-size effects might play a role in the symmetry breaking process. Finally, in Sec. II(d) the example of ultrathin films grown on substrates with a gradient in atomic step density is considered, where the steps act as the symmetry breaking features that control the magnetic state of the system.

\section{Discussion of Results}

\section{(a) Two dimensional magnetic phase transition}

In a two-dimension (2D) system, it is well known from the Mermin-Wagner theorem ${ }^{1}$ that a Heisenberg system does not have long-range magnetic order at finite temperature. Since a real experimental system always possesses magnetic anisotropy energy, an interesting question is what is the effect of the magnetic anisotropy on the magnetic long-range order and the magnetic phase transition? The simplest form of magnetic anisotropy is uniaxial anisotropy for which there is a special direction for the electron spins. Uniaxial anisotropy cannot exist in bulk materials with cubic symmetry, but can exist at a thin-film surface due to the broken 
translational symmetry in the surface normal direction. The next higher-order term in the magnetic anisotropy is proportional to the fourth power of the magnetization and is usually referred to as the volume anisotropy. A 2D magnetic thin film with cubic symmetry should belong to one of two classes: (1) if a uniaxial anisotropy is present, then the system is described by a Heisenberg model plus the uniaxial anisotropy; or (2) if the magnetization is in the film plane but there is no in-plane uniaxial anisotropy, then the system is described by an XY model plus a volume anisotropy. For the first film class, Bander and Mills showed ${ }^{2}$ that the uniaxial anisotropy stabilizes the magnetic long-range order and makes the magnetic phase transition 2D Ising-like. For the second film class, however, José et al. showed ${ }^{3}$ that the quadratic anisotropy will result in a non-universal transition.

Experimental investigations of $2 \mathrm{D}$ magnetic phase transitions usually measure the temperature dependence of the magnetization to determine a critical exponent $\beta$ by the power law: $\mathrm{M}(\mathrm{T}) \sim\left(1-\mathrm{T} / \mathrm{T}_{\mathrm{C}}\right)^{\beta}$. Examples of systems studied include $\mathrm{Fe} / \mathrm{Au}(100),{ }^{4} \mathrm{Fe} / \mathrm{Pd}(100),{ }^{5}$ $\mathrm{Fe} / \mathrm{Ag}(111),{ }^{6} \mathrm{Co} / \mathrm{Cu}(111),{ }^{7} \mathrm{Ni} / \mathrm{Cu}(100),{ }^{8} \mathrm{Ni} / \mathrm{Cu}(111),{ }^{9} \mathrm{Ni} / \mathrm{W}(110),{ }^{10}$ and $\mathrm{Tb} / \mathrm{W}(110),{ }^{11}$ It is interesting to note that the $\beta$ values determined usually fall into two groups: one has $\beta$ close to the $2 \mathrm{D}$ Ising value of $1 / 8$, and the other has $\beta$ close to the value $1 / 4$. Almost all films in the $\beta \approx 1 / 8$ group have either a perpendicular magnetic anisotropy or an in-plane uniaxial anisotropy, and therefore, belong to the first class of films mentioned above. Films in the $\beta \approx 1 / 4$ group belong without exception, according to our knowledge, to the second film class. In this section, we present two examples of the first film class. In Sec. 3 we discuss an example illustrating the second class.

The two systems of the first type studied are $\mathrm{Fe} / \mathrm{Ag}(100)$ and $\mathrm{Fe}(110) / \mathrm{Ag}(111)$. $\mathrm{Fe} / \mathrm{Ag}(100)$ has a perpendicular easy magnetization axis in the ultrathin regime $^{12}$, and $\mathrm{Fe}(110) / \mathrm{Ag}(111)$ has in-plane magnetization with an in-plane surface anisotropy. In addition, the Fe/Ag system has two advantages: (i) $\mathrm{Fe}$ and $\mathrm{Ag}$ are immiscible, which inhibits alloying and interdiffusion at the interface, and yields a thermally reversible magnetization, and (ii) the 
$d$-electrons of Fe hybridize weakly with the $s p$-valence electrons of $\mathrm{Ag},{ }^{13}$ so that ultrathin films of Fe on $\mathrm{Ag}$ form an almost ideal 2D ferromagnetic system.

Sample preparation is always important in surface science and is presented briefly here. The $\mathrm{Ag}(100)$ substrate was mechanically polished $\left(\mathrm{Al}_{2} \mathrm{O}_{3}\right.$ powder) to a $0.05 \mu$ finish, and then chemically polished with a cotton swab soaked in a solution of saturated chromic acid and $\sim 1 \%$ hydrochloric acid, and then rinsed in water. ${ }^{14}$ The substrate was then ultrasonically cleaned in methanol before its introduction into the ultra-high vacuum (UHV) chamber. Finally the surface was cleaned in UHV by cycles of $2 \mathrm{keV} \mathrm{Ar}+$ ion sputtering and annealing at $\sim 700{ }^{\circ} \mathrm{C}$. The $\mathrm{Ag}(111)$ substrate was prepared by depositing $\sim 1000 \AA$ of $\mathrm{Ag}$ onto mica held at $450 \mathrm{~K}$. The Fe film was grown onto the $\mathrm{Ag}(100)$ substrate at room temperature, and on $\mathrm{Ag}(111)$ at $450 \mathrm{~K}$. The evaporation rate was typically $\sim 0.6 \AA / \mathrm{min}$ and was measured by a quartz thickness monitor. The magnetic properties of the films were studied in situ by means of the surface magneto-optic Kerr effect (SMOKE) technique using a He-Ne laser as the light source. The magnetic field was applied either perpendicular (polar) or parallel (longitudinal) to the film plane $^{15}$ to generate magnetic hysteresis loops.

Figure 1 shows hysteresis loops taken at different temperatures for a 2.5 monolayer (ML) Fe film grown on $\mathrm{Ag}(100)$. At low temperature, the loop is relatively square and has almost full remanence, indicating a well established spontaneous magnetization. As the temperature is increased, the remanence decreases and finally disappears above the Curie temperature $T_{C}$. To study the magnetic phase transition, we recorded the magnetic remanence for two Fe films (2.5 and $2.7 \mathrm{ML})$ as a function of temperature near $\mathrm{T}_{\mathrm{C}}$. The results are plotted in Fig. 2(a). It is obvious that the magnetization undergoes a second-order phase transition upon increasing temperature. Since the Kerr intensity is proportional to the magnetization, $M$, we can fit our data to the power law $M(T) \sim\left(1-T / T_{C}\right)^{\beta}$ in the vicinity of Curie temperature with $T_{C}$ and the effective value of $\beta$ as fitting parameters. To explore the power law further, we made a log-log plot of the remanent magnetization and reduced temperature 1- 
T/T [Fig. 2(b)]. The straight line in Fig. 2(b) depicts the power law, and the slope yields $\beta=0.124 \pm 0.002$ in good agreement with the $2 \mathrm{D}$ Ising critical value of $1 / 8$. The fitting results give $\mathrm{T}_{\mathrm{C}}$ values $\sim 3-5 \%$ that are lower than the temperature at which the remanence vanishes. The existence of a tail above $T_{C}$ is frequently encountered in magnetic film studies and is attributed to a finite-size effect. ${ }^{16}$ This effect limits the divergence of the correlation length and results in a tail above $\mathrm{T}_{C}$ by an amount $\Delta \mathrm{T}_{\mathrm{C}} \sim a / l$, where $a$ and $l$ are the lattice parameter and the lateral coherence length of the film, respectively. Therefore a 3-5\% tail above $\mathrm{T}_{\mathrm{C}}$ implies a finite size of $l \sim 100 \AA$, which is in accord with expectation for a typical terrace size for a metallic surface. In addition, the fact that $l$ is much greater than the Fe film thickness ensures the $2 \mathrm{D}$ nature of the magnetic phase transition. Similar results were also obtained for the $\mathrm{Fe}(110) / \mathrm{Ag}(111)$ system [Fig. 3(a) and 3(b)]. The slope of the log-log plot in that case yields $\beta=0.137$, again very close to the $2 \mathrm{D}$ Ising value of $1 / 8$. Therefore, we can conclude that the magnetic phase transition in thin films with uniaxial magnetic anisotropy belongs to the $2 \mathrm{D}$ Ising universality class.

\section{(b) Spin reorientation transition}

For a thin film with perpendicular magnetic surface anisotropy, the magnetization can be perpendicular to the film below a critical film thickness $\mathrm{d}_{\mathrm{R}}$ or below a critical temperature $T_{R}$, but parallel to the film surface above $d_{R}$ or $T_{R}$. This directional switching of the magnetization is called a spin reorientation transition (SRT). The driving force for the 2D SRT is the competition between the perpendicular surface anisotropy, which is inversely proportional to the film thickness, and the volume-type shape anisotropy, which is independent of film thickness. At the critical point $d_{R}$ or $T_{R}$, these two types of anisotropies compensate so that the SRT occurs. Research on the 2D SRT was originally motivated again by the MerminWagner theorem. If the uniaxial magnetic anisotropy stabilizes the magnetic long-range order (LRO) in a 2D Heisenberg system, an interesting question is what happens to the LRO at the SRT point where the effective anisotropy vanishes due to cancellation between the surface and 
shape contributions? Early theoretical studies ${ }^{17}$ suggested that in the vicinity of $T_{R}$ there is a region in temperature $\Delta T_{R}$ wherein the magnetic LRO is lost. Experimentally, Pappas et al. investigated $\mathrm{Fe} / \mathrm{Cu}(100)^{18}$ and $\mathrm{Fe} / \mathrm{Ag}(100)^{19}$ by means of spin-polarized secondary-electron spectroscopy and, indeed, reported the existence of such a temperature interval, but their value of $\Delta T_{R}$ was an order of magnitude larger than predicted. We investigated the $\mathrm{Fe} / \mathrm{Ag}(100)$ system using SMOKE. Our sensitivity permitted us to identify detailed features within a pseudo gap region encompassing $\Delta T_{R}$.

The Fe film for this example was grown into a wedge shape (with slopes of 0.2-0.5 $\mathrm{ML} / \mathrm{mm}$ ) with the gradient along the [001]. Wedge structures readily permit thickness dependences to be monitored in a controlled and continuous fashion. The method used to create the wedge was to translate the Ag substrate behind a knife-edge shutter during film growth. The slope of the wedge is controlled by the evaporation rate and by the substrate translational speed. The magnetic properties of the films were investigated in situ by means of SMOKE measurements with the external magnetic field either perpendicular (polar) or parallel (longitudinal) to the film plane. ${ }^{20}$ The laser beam was focused onto the sample. to a spot size of $\sim 0.2 \mathrm{~mm}$. Thus, the thickness variation due to the wedge slope is only $\sim 0.04-0.1 \mathrm{ML}$ within the laser spot; so the thickness within the laser spot is virtually uniform.

SMOKE results are shown in Fig. 4. Figure 4(a) illustrates the perpendicular magnetic remanence $\left(M_{\perp}\right)$ determined from the polar signal, and the parallel remanence $\left(M_{/ /}\right)$determined from the longitudinal signal, for a 6-ML Fe film at different temperatures. Figure 4(b) contains data at room temperature for different Fe film thicknesses. The SRT can be clearly seen as a function of both temperature and thickness. At low temperature or low thickness, $M_{\perp}$ remains at its saturation value. At high temperature or large thickness, $M_{/ /}$retains its saturation value. In the SRT region ( $\Delta \mathrm{T}$ and $\Delta \mathrm{d}$ in Fig. 4 ), $\mathrm{M}$ is greatly suppressed from its saturation value. This region is similar to that observed in $\mathrm{Fe} / \mathrm{Cu}(100)$ and $\mathrm{Fe} / \mathrm{Ag}(100)$ by Pappas et al. 8,9 But the significant difference is that $\mathrm{M}$ is not zero in this region. We identify this region (and that 
of Pappas et al. ${ }^{9}$ by implication) as not being associated with a loss of LRO, but as a pseudo gap which possesses a complex magnetic structure.

The formation of magnetic domains within the psudo-gap region provides a possible explanation for the suppression of $M$ but without a loss of magnetic LRO. Yafet and Gyorgy recognized that in a 2D Heisenberg system with uniaxial anisotropy the formation of stripe domains is favored over the single domain structure. The mechanism for the formation of the strip domains involves the competition between the dipole interaction and the short-range exchange interaction ${ }^{21}$. The dipole interaction can be decomposed in short- and long-range parts; the short-range part gives rise to the familiar shape anisotropy. The size of the stripe domains depends critically on the strength of the long-range part. Only near the SRT, where the magnetic surface anisotropy compensates the short-range part of the dipole interaction (shape anisotropy), can micron-size strip domains be formed as a result of the long-range part of the dipole interaction. Erickson and Mills ${ }^{22}$ reached a similar conclusion by examining the behavior of $2 \mathrm{D}$ spin-wave excitations under the influence of the dipole interaction. Stripe domains are a 1D ordered state which should not be stable against quantum fluctuations. Indeed, Kashuba and Pokrovsky ${ }^{23}$ found that stripe domain structure is equivalent to a $2 \mathrm{D}$ liquid crystal system which has orientation order but no spatial order. Allenspach and Bischof $^{24}$ applied the SEMPA technique to the $\mathrm{Fe} / \mathrm{Cu}(100)$ system and observed that the single domain structure of the film breaks into stripe domains $(\sim 1 \mu \mathrm{m}$ size $)$ in the SRT region, and the the stripe domains are randomly distributed in space, but with orientation order.

\section{(c) Manganite intergrowths}

Doped manganite oxides have recently gained attention due to interest in the property of colossal magnetoresistance (CMR). CMR occurs in many of these materials in the vicinity of their Curie transition, which is also where they undergo a metal-insulator transition. At low temperature they are ferromagnetic metals and at elevated temperatures they are polaronic 
insulators. While many of these manganites are bulk perovskite-based crystals with relatively high Curie temperatures, i.e. $\mathrm{La}_{1-\mathrm{x}} \mathrm{Sr}_{\mathbf{x}} \mathrm{MnO}_{3}$, some of the more intriguing challenges arise in naturally layered variants of the Ruddlesden-Popper series which have substantially lower Curie temperatures for similar doping levels, such as in the $\mathrm{SrO}\left(\mathrm{La}_{1-\mathrm{x}} \mathrm{Sr}_{\mathrm{x}} \mathrm{MnO}_{3}\right)_{2}$ bilayer structure which is separated by SrO barrier layers. In these layered systems, similar to naturally layered cuprate and ruthenate structures, occasional stacking faults can introduce inclusions also termed intergrowths which have larger values of $n$ than the host material. The value $n=5$ intergrowths have been identified in the bilayer system referred to above. ${ }^{25}$ Such a system is of particular interest to the surface magnetism community because in the temperature interval that is above the Curie temperature of the host material but below the Curie temperature of the intergrowth, an atomically flat $2 \mathrm{D}$ ferromagetic inclusion is present. In the previous section it was already noted that a terrace length of $100 \AA$ is a typically coherence value for a $2 \mathrm{D}$ film that is artificially grown. The intergrowth can have significantly superior lateral smoothness and coherency.

In Fig. 5 we examine the magnetization of $\operatorname{SrO}\left(\mathrm{La}_{1-x} \mathrm{Sr}_{x} \mathrm{MnO}_{3}\right)_{2}$ for $\mathrm{x}=0.4$ in the vicinity of the Curie transition associated with the intergrowths. The intergrowth magnetization represents only a fraction of $1 \%$ of the total magnetization of that the host material develops below its bulk Curie temperature. The easy axis is in the plane of the layering of the structure. The effective magnetization exponent $\beta$ is $0.25 \pm 0.02$, in accord for expectation for the second type of film class introduced above. This value has been identified with 2D systems for which the magnetization is in plane, but for which there is no uniaxial anisotropy present. This $\beta=0.25$ behavior does not correspond theoretically to that for any know universality class, but a value of $\beta$ of $\sim 0.231$ has been determined recently for the finite-size $2 \mathrm{D} \mathrm{XY}$ model. ${ }^{26}$ The finite size in that case is the symmetry-breaking feature that permits magnetic LRO to be established at finite temperature. It is intriguing to associate the experimental intergrowth value of 0.25 with this theoretical model, but a word of caution must be inserted. The experimental transition is 
quite significantly broadened (see Fig. 5 inset) as might be expected from, say, compositional variations. Thus, we leave this topic with the thought that verifying theory always requires dialogue. Our point is not to claim verification, but to open a creative dialogue.

\section{(d) Step-induced magnetic anisotropy}

The magnetic anisotropy energy originates from the spin-orbit interaction ${ }^{27}$ and can be expressed as:

$$
E=\sum_{\alpha \beta} K_{\alpha \beta} u_{\alpha} u_{\beta}+\sum_{\alpha \beta \gamma \delta} K_{\alpha \beta \gamma \delta} u_{\alpha} u_{\beta} u_{\gamma} u_{\delta}+\cdots,
$$

where $u_{\alpha}$ is the $\alpha$-th component of the magnetization vector, and $K_{\alpha \beta}$ and $K_{\alpha \beta \gamma \delta}$ are the anisotropy constants. Although the anisotropy constants depend in detail on the band structure, the functional form of the anisotropy in eqn. (1) depends only on the lattice symmetry. For example, the anisotropy energy in a lattice with cubic symmetry must take the form:

$$
E=K\left(u_{x}^{2} u_{y}^{2}+u_{y}^{2} u_{z}^{2}+u_{z}^{2} u_{x}^{2}\right)
$$

regardless of the value of the anisotropy constant $\mathrm{K}$. If the translation symmetry of the lattice is broken along one direction, then a square-term uniaxial anisotropy will be generated. Since the translation symmetry is usually broken at the surface of a crystal, the square term anisotropy is often termed the surface anisotropy, and takes the following form.

$$
E_{s}=K_{s} \mathrm{u}_{z}^{2}+K_{S P} \mathrm{u}_{x}^{2}
$$

where $\mathrm{z}$ is the surface normal direction and $\mathrm{x}$ is one principal axis in the plane of the surface. Based on a symmetry argument, it can be shown that $K_{\mathrm{sp}}$ will vanish if the surface normal is an $\mathrm{n}$-fold rotation axis with $\mathrm{n}>2 .^{28}$ Thus the in-plane surface anisotropy cannot exist in triangular or square 2D lattices. Understanding the functional form of the magnetic anisotropy and its relation to the lattice symmetry is a very important issue. There is experimental evidence for the existence of an intrinsic relationship between the magnetic anisotropies along different crystalline orientations. ${ }^{29}$ There have also been efforts ${ }^{30,31}$ to construct functional forms for the 
magnetic anisotropy under different circumstances. Knowledge about the role of symmetrybreaking in the magnetic anisotropy is very limited. It is not even clear to what extent Néel's pair-bonding model ${ }^{32}$ correctly describes the functional form of the magnetic anisotropy.

To gain a deeper understanding of the magnetic anisotropy, we used stepped surfaces to explore the role of lattice symmetry-breaking. For a (100) surface of an fcc or bcc lattice, the four-fold rotation symmetry of the surface prohibits the existence of an in-plane, uniaxial magnetic anisotropy. Well-defined nanometer-sized atomic steps on the (100) surface, however, could break the four-fold rotation symmetry at step edges and induce a uniaxial anisotropy within the film plane. Since the degree of symmetry-breaking depends on stepdensity, an investigating of the relation between step-induced anisotropy and step density is needed. Experimentally, atomic steps can be created on a surface by cutting a crystal with a small vicinal angle. If the lattice constant in the normal direction is $a$ and the vicinal angle is $\alpha$, the step terrace length is $\mathrm{L} \sim a / \alpha$. Thus, a few degrees of vicinal angle creates nanometer-sized atomic steps. In fact, this type of step-induced magnetic anisotropy was reported in several systems. ${ }^{33}, 34,35,36$ In these studies, the vicinal angle was fixed so that the relation between the step-induced anisotropy and step density was not explored. To obtain such a relation, many samples with different vicinal angles would be needed. However, it is very difficult experimentally to prepare multiple samples under identical conditions. To overcome this difficulty we used a curved substrate where the curved shape provides a continuous change of the vicinal angle. The magnetic properties of the films were measured by the SMOKE technique. As the SMOKE laser beam scans across the sample to measure the hysteresis loops, its reflection angle simultaneously determines the local vicinal angles at different positions along the substrate. Therefore, the relation between the step-induced anisotropy and step density can be obtained systematically from a single curved substrate.

We discuss results for three representative systems: $\mathrm{Fe} / \mathrm{W}(001), \mathrm{Co} / \mathrm{Cu}(001)$ and $\mathrm{Fe} / \mathrm{Pd}(001)$. In $\mathrm{Fe} / \mathrm{W}$ and $\mathrm{Fe} / \mathrm{Pd}$, the steps are parallel to the [100] direction of the $\mathrm{Fe}$. In 
$\mathrm{Co} / \mathrm{Cu}$, the steps are parallel to the $[1 \overline{1} 0]$ direction of the Co. The SMOKE laser beam of $\sim 0.3$ $\mathrm{mm}$ diameter would cover $\sim 0.7^{\circ}$ of vicinal angle, but a narrow slit placed in the reflection path improved the angular resolution to $\sim 0.2^{\circ}$. The magnetization is in the film plane for all three systems, thus only longitudinal hysteresis loops are reported. Figure 6 shows hysteresis loops for a 2-ML Fe film grown on stepped $\mathrm{W}(001)$ at $4.7^{\circ}$ vicinal angle. The stepped Fe film shows a square loop with $\sim 100 \%$ remanence for the magnetic field parallel to the step edges, and two split subloops with zero remanence for the field perpendicular to the step edges. This behavior indicates that the atomic steps indeed induce an in-plane, uniaxial magnetic anisotropy with the easy magnetization axis perpendicular to the step edges. The easy axis of the stepinduced anisotropy depends on the physical system. While the $\mathrm{Fe} / \mathrm{W}(001)^{37}$ and $\mathrm{Fe} / \mathrm{Pd}(001)^{38}$ systems have their easy axis perpendicular to the step edges, the $\mathrm{Co} / \mathrm{Cu}(001)^{39}$ and $\mathrm{Fe} / \mathrm{Ag}(001)^{40}$ systems have their easy axis parallel to the step edges. Nevertheless, the splitting field $\mathrm{H}_{\mathrm{S}}$, as defined in Fig. 6 for the hard-axis loop, is proportional to the strength of the step-induced anisotropy. Figure 7 shows the relation between $H_{s}$ and the vicinal angle $\alpha$ (which is proportional to the step density) for the three systems. Fitting $\mathrm{H}_{\mathrm{s}} \sim \alpha^{n}$ (the solid lines in Fig. 7) yields an exponent $n=2$ for the $\mathrm{Fe} / \mathrm{W}$ system, but $n=1$ for the $\mathrm{Co} / \mathrm{Cu}$ and $\mathrm{Fe} / \mathrm{Pd}$ systems. To understand why there are different dependence of $\mathrm{H}_{S}$ on $\alpha$ from different systems, one has to examine how the symmetry of the lattice at the step edges is broken for bcc and fcc structures. In the Néel pair-bonding model ${ }^{41}$, the magnetic anisotropy is generated by the nearest-neighbor bonds. For bcc and fcc lattices, there is no uniaxial anisotropy because contributions from all nearest-neighbor bonds cancel out the square-term anisotropy. At the step edges, however, the missing atoms break this cancellation so that uniaxial anisotropy will be manifest.

For a bcc lattice with steps parallel to the [100] direction, the anisotropy due to the missing atoms should have the form $E_{a}=-(K / L) u_{\eta} u_{\zeta}$, where $\mathrm{L}$ is the terrace length, $\mathbf{u}$ is the unit vector of the magnetization $\mathbf{M}$, and $\xi, \eta$ and $\zeta$ are the [100], [010] and [001] axes, 
respectively. For an fcc lattice with steps parallel to the [110] direction, the anisotropy due to the missing atoms has the form $E_{a}=-\frac{K}{L}\left(2 u_{\eta}^{2}+3 u_{\zeta}^{2}+2 \sqrt{2} u_{\eta} u_{\xi}\right)$, where $\xi=[110]$, $\eta=[110]$ and $\zeta=[001]$. Note that the normal direction ( $\mathrm{z}$ axis) of the stepped surface makes a small vicinal angle $\alpha$ to the [001] axis (so that $\alpha \approx 1 / L$ ). The crystal $\xi \eta \zeta$-frame of reference has to be transformed from $u_{\xi}=u_{x}, u_{\eta}=u_{y} \cos \alpha+u_{z} \sin \alpha$, and $u_{\xi}=-u_{y} \sin \alpha+u_{z} \cos \alpha$, into to the film xyz-frame with the $x$ - and $y$-axes in the film plane parallel and perpendicular to the step edges, respectively. The anisotropy (for small $\alpha$ ) transforms to $E_{a}=-K\left(\alpha u_{y} u_{z}-\alpha^{2} u_{y}^{2}+\alpha^{2} u_{z}^{2}\right)$ for a bcc lattice, and $E_{a}=-K\left(2 \alpha u_{y}^{2}+3 \alpha u_{z}^{2}+2 \sqrt{2} \alpha u_{y} u_{z}\right)$ for an fcc lattice. Therefore the in-plane, step-induced anisotropy $\left(u_{z}=0\right)$ is $E_{a}=K \alpha^{2} u_{y}^{2}$ for the bcc case, and $E_{a}=-2 K \alpha u_{y}^{2}$ for fcc. This provides an explanation for why $\mathrm{Fe} / \mathrm{W}$ and $\mathrm{Co} / \mathrm{Cu}$ exhibit quadratic and linear $\alpha$-dependences, respectively, for their step-induced anisotropies. The most interesting result is the linear dependence in Fe/Pd system. Fe has a bcc structure but $\mathrm{Pd}$ has an fcc structure. It was shown that the Pd at the interface of Fe/Pd is ferromagnetic due to the Fe spin polarization. ${ }^{42}, 43$ Since Pd has a much stronger spin-orbit interaction than $\mathrm{Fe}$, the Pd is expected to dominate the magnetic anisotropy in the Fe/Pd system. We believe that is why the Fe/stepped $\operatorname{Pd}(001)$ system exhibits an $\alpha$-linear dependence of the step-induced anisotropy as for an fcc lattice.

\section{Afterword}

The present work highlighted examples of magnetic surface and interfacial phenomena that involves symmetry breaking as a key element. The symmetry breaking either permits phase transitions to occur at finite temperature or gives rise to magnetic anisotropies that govern the physical characteristics of the system. Diverse examples are used to illustrate specific effects. In each case the examples are taken from prior work by the present authors. The Curie transition and the 2D spin reorientation transition are considered for ferromagnetic overlayers and wedge-shaped structures in the presence of surface magnetic anisotropy. The 
magnetization of an unusual 2D inclusion in the bulk of a naturally layered manganite crystal is presented. Step-induced magnetic anisotropy is explored on novel curved crystal substrates that possess a continuous gradient in atomic step density. These topics are meant to stimulate awareness of the opportunities to study different symmetry crossings in 2D magnetic systems.

\section{Acknowledgments}

We thank the many colleagues that we have worked with on the projects cited in the reference list. Without them this work would not be possible. This work was financially supported by the Office of Basic Energy Sciences - Materials Sciences of the United States Department of Energy under Contract No. W-31-109-ENG-38 (at Argonne) and DE-AC03-76SF00098 (at Berkeley) and by National Science Foundation Contract No. DMR-9805222 (at Berkeley). 


\section{Figure Captions:}

Fig. 1: Hysteresis loops of a $2.5 \mathrm{ML}$ Fe film on $\mathrm{Ag}(001)$ at different temperatures.

Fig. 2. (a) The magnetic remanences of a $2.5 \mathrm{ML}$ and a $2.7 \mathrm{ML}$ Fe films on $\mathrm{Ag}(100)$ as a function of temperature. (b) Log-log plot of $M v s$ 1-T/TC. The solid lines depict the theoretical fitting of a power law: $M(T) \sim\left(1-T / T_{C}\right)^{\beta}$, where $T_{C}$ and $\beta$ are fitting parameters for the Curie temperature and the critical exponent, respectively.

Fig.3. (a) The magnetic remanences of $1.8,1.9$, and $2.0 \mathrm{ML} \mathrm{Fe}$ films on $\mathrm{Ag}(111)$ as a function of temperature. (b) Log-log plot of $M v s 1-T / T_{C}$. The solid lines depict the theoretical fitting of a power law: $M(T) \sim\left(1-T / T_{C}\right)^{\beta}$, where $T_{C}$ and $\beta$ are fitting parameters for the Curie temperature and the critical exponent, respectively.

Fig. 4. The perpendicular and parallel components of the remanent magnetization (a) for a 6.0 ML Fe on $\mathrm{Ag}(100)$ vs $\mathrm{T}$, and (b) for different Fe film thicknesses at room temperature. $\Delta \mathrm{T}$ and $\Delta \mathrm{d}$ define the pseudo gap of the spin-reorientation transition.

Fig. 5 The magnetization for a trace 2D intergrowth inclusion in a bulk single crystal of the bilayer manganite $\mathrm{SrO}\left(\mathrm{La}_{1-\mathrm{x}} \mathrm{Sr}_{\mathbf{x}} \mathrm{MnO}_{3}\right)_{2}$ for $\mathrm{x}=0.4$.

Fig.6. Hysteresis loops for a 2-ML Fe film grown on a $4.7^{\circ}$ miscut stepped W(001) surface. The square loop is for $\mathrm{H}$ perpendicular to the step edges and the split loop is for $\mathrm{H}$ parallel to the step edges.

Fig. 7. $\mathrm{H}_{\mathrm{S}}$ (from Fig. 6) vs. vicinal angle $\alpha$ for (a) $\mathrm{Fe} / \mathrm{W}(001)$, (b) $\mathrm{Co} / \mathrm{Cu}(001)$, and (c) $\mathrm{Fe} / \mathrm{Pd}(001)$. The solid lines are results of a power-law fitting yielding a quadratic relation between $\mathrm{H}_{\mathrm{S}}$ and $\alpha$ for the $\mathrm{Fe} / \mathrm{W}$ system, but a linear relation for the $\mathrm{Co} / \mathrm{Cu}$ and Fe/Pd systems. 


\section{References:}

1. M.D. Mermin and H Wagner, Phys. Rev. Lett. 17, 1133 (1966).

2. M. Bander and D. L. Mills, Phys. Rev. B 38, 12015 (1988).

3. J. V. José, L. P. Kadanoff, S. Kirkpatrick, and D R. Nelson, Phys. Rev. B. 16, 1217 (1977).

4. W. Dürr et al.,Phys. Rev. Lett. 62, 206 (1989).

5. C. Liu and S. D. Bader, J. Appl. Phys. 67, 5758 (1990).

6. Z. Q. Qiu, J. Pearson, and S. D. Bader, Phys. Rev. Lett. 67, 1646 (1991).

7. J. Kohlhepp, H. J. Elmers, S. Cordes, and U. Gradmann, Phys. Rev. B 45, 12287 (1992).

8. F. Huang, G. J. Mankey, M. T. Kief, and R. F. Willis, J. Appl. Phys. 736760 (1993).

9. C. A. Ballentine et al., Phys. Rev. B 41, 10175 (1990).

10. Y. Li and K. Baberschke, Phys. Rev. Lett. 68, 1208 (1992).

11. C. Rau, Appl. Phys. A 49, 579 (1989).

12. B.T. Jonker, K.-H. Walker, E. Kisker, G.A. Prinz, and C. Carbone, Phys. Rev. Lett. 57, 142 (1986); B. Heinrich, K. B. Urquhart, A. S. Arrott, J. F. Cochran, K. Myrtle, and S. T. Purcell, ibid, 59, 1756 (1987); M. Stampanoni, A. Vaterlaus, M. Aeschlimann, and F. Meier, ibid, 59, 2483 (1987); J. Arayapochet, C. A. Ballentine, and J. L. Erskine, Phys. Rev. B. 38, 7846 (1988).

13. C. L. Fu, A. J. Freeman, and T. Oguchi, Phys. Rev. Lett. 54, 2700 (1985).

14. N. Q. Lam, S. J. Rothman, and L. J. Nowicki, J. Electro-Chem. Soc. 119, 715 (1972).

15. J. Zak, E.R. Moog, C. Liu, and S.D. Bader, J. Magn. Magn. Mater. 89, 107 (1990).

16. D. P. Landau, Phys. Rev. B 13, 2997 (1976).

17. D. Pecia and V.L. Pokrovsky, Phy. Rev. Lett. 65, 2599 (1990).

18. D.P. Pappas, K.-P. Kämper, and H. Hopster, Phys. Rev. Lett. 64, 3179 (1990).

19. D.P. Pappas, C.R. Brundle, and H. Hopster, Phys. Rev. B 45, 8169 (1992)

20. J. Zak, E.R. Moog, C. Liu, and S.D. Bader, J. Magn. Magn. Mater. 89, 107 (1990).

21. Y Yafet and E. M. Gyorgy, Phys. Rev. B 38, 9145 (1988). 
22. R. P. Erickson and D. L. Mills, Phys. Rev. B 46, 861 (1992).

23. A. Kashuba and V. L. Pokrovsky, Phys. Rev. Lett. 70, 3155 (1993); Phys. Rev. B 48, 10335 (1993).

24. R. Allenspach and A. Bischof, Phys. Rev. Lett. 69, 3385 (1992).

2s . S.D. Bader, D. J. Miller, J. F. Mitchell. R. M. Osgood III, and J. S. Jiang, J. Appl. Phys. 83, 6385 (1998).

26. S.T Bramwell and P.C.W. Holdsworth, J. Phys. Condensed Matter 5, L53 (1993).

27. J. H. van Vleck, Phys. Rev. 52, 1178 (1937).

28. U. Gradmann, J. Korecki, and G. Waller, Appl. Phys. A 39, 101-108 (1986).

29. B. N. Engel, C. D. England, R. A. Van Leeuwen, M. H. Wiedmann, and C. M. Falco, Phys. Rev. Lett. 67, $1910(1991)$.

30. R. H. Vicotra and J. M. MacLaren, Phys. Rev. B 47, 11583 (1993).

31. D. S. Chuang, C. A. Ballentine, and R. C. O'Handley, Phys. Rev. B 49, 15084 (1994).

32. L. Néel, J. Phys. Radium 15, 225 (1954).

33. B. Heinrich, S. T. Purcell, J. R. Dutcher, K. B. Urquart, J. F. Cochran, and A. S. Arrott, Phys. Rev. B 38, 12879 (1988).

34. A. Berger, U. Linke, and H. P. Oepen, Phys. Rev. Lett. 68, 839 (1992).

35. W. Weber, C. H. Back, A. Bischof, Ch. Würsch, and R. Allenspach, Phys. Rev. Lett. 76, 1940 (1996).

36. J. Chen and J. Erskine, Phys. Rev. Lett. 68, 1212 (1992).

37. Hyuk J. Choi, Z. Q. Qiu, J. Pearson, S. J. Jiang, D. Li, and S. D. Bader, Phys. Rev. B 57, R12713 (1998).

38. Hyuk J. Choi, R. K. Kawakami, Ernesto J. Escorcia-Aparicio, and Z. Q. Qiu, J. Pearson, J. S. Jiang, Dongqi Li, and S. D. Bader, submitted.

39. R. K. Kawakami, M. O. Bowen, Hyuk J, Choi, Ernesto J. Escorcia-Aparicio, and Z. Q. Qiu, Phys. Rev. B 58, R5924 (1998).

40. R. K. Kawakami, Ernesto J. Escorcia-Aparicio, and Z. Q. Qiu, Phys. Rev. Lett. 77, 2570 (1996). 
4!. L. Néel, J. Phys. Radium 15, 225 (1954).

42. O. Rader, E. Vescovo, J. Redinger, S. Blügel, C. Carbone, W. Eberhardt, and W Gudat, Phys. Rev. Lett. 72, 2247 (1994).

43. C. Liu and S. D. Bader, Phys. Rev. B 44, 2205 (1991). 


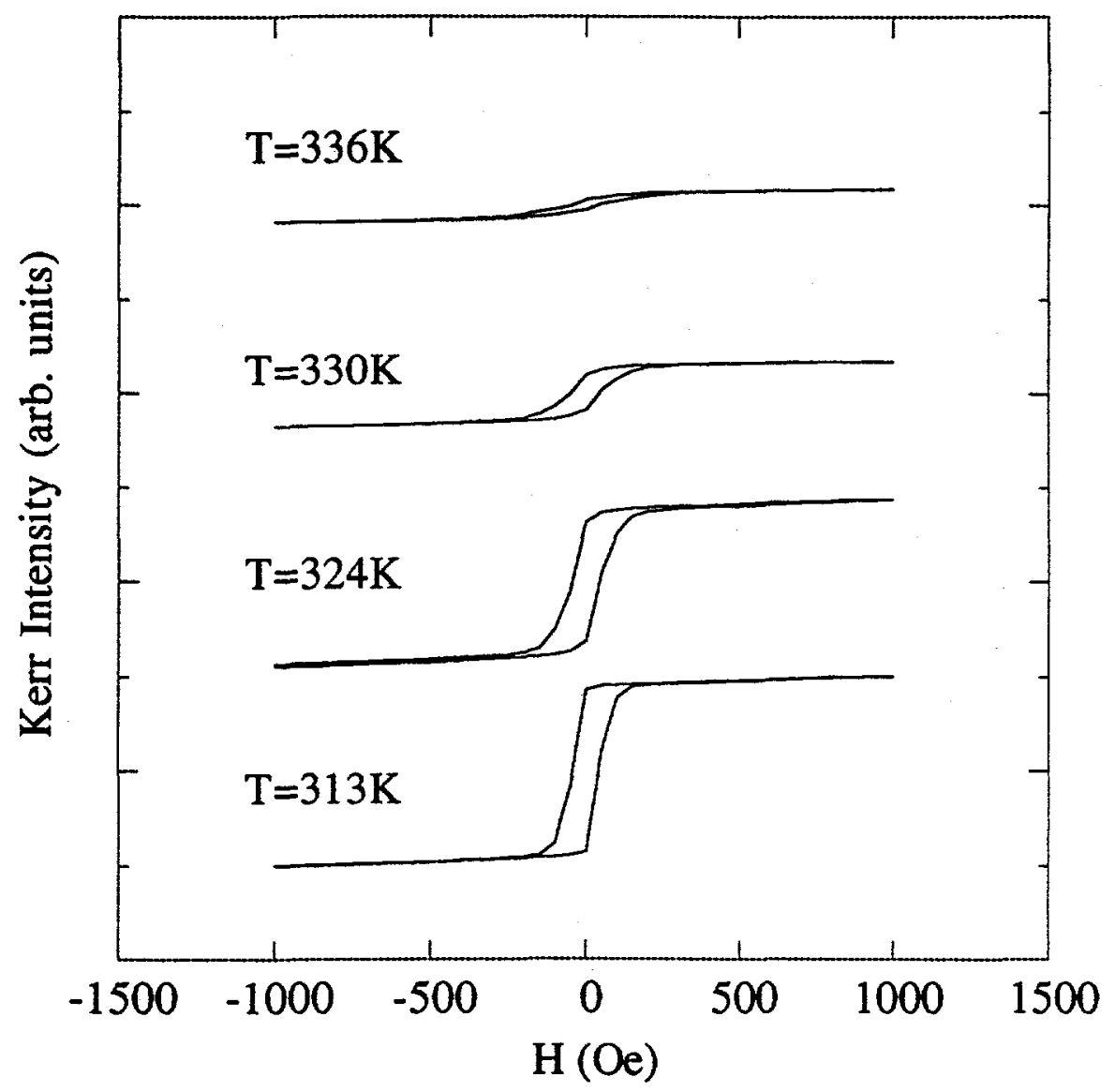




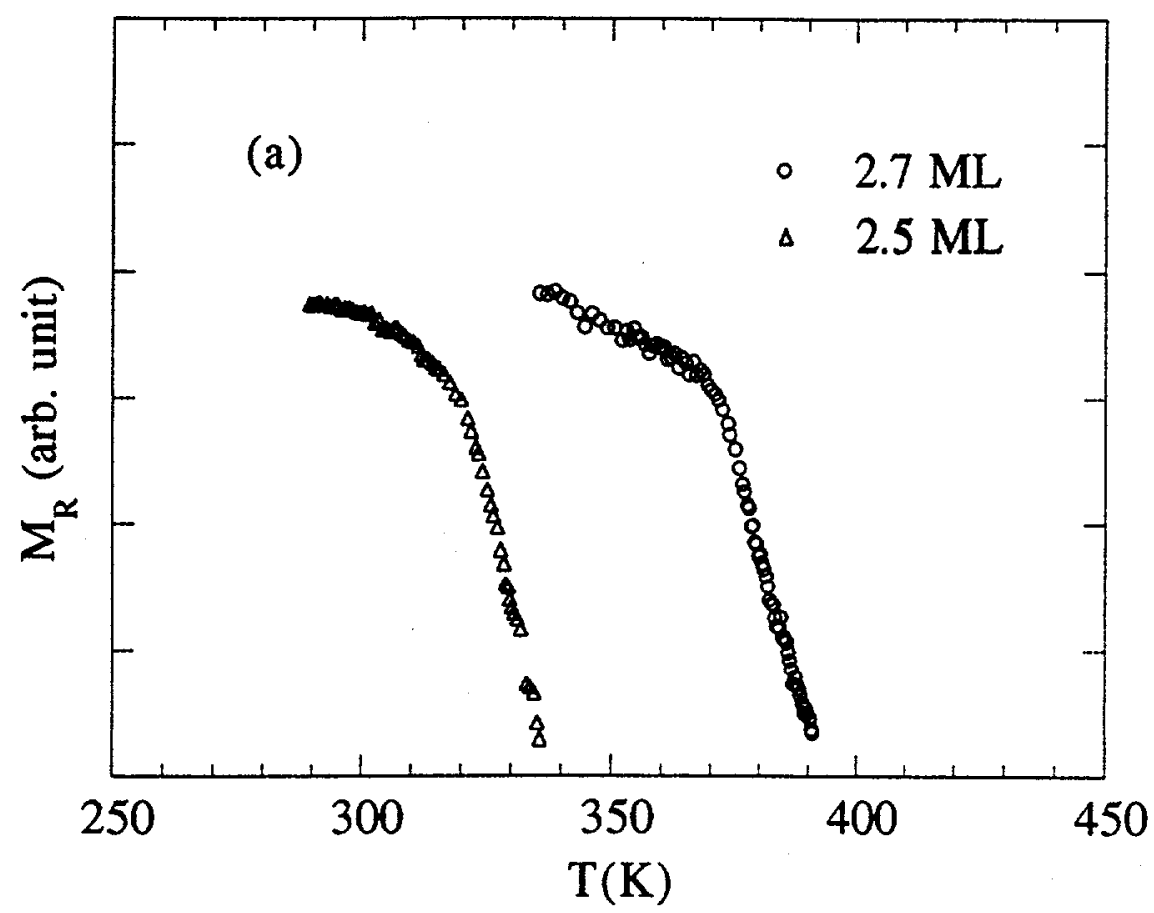




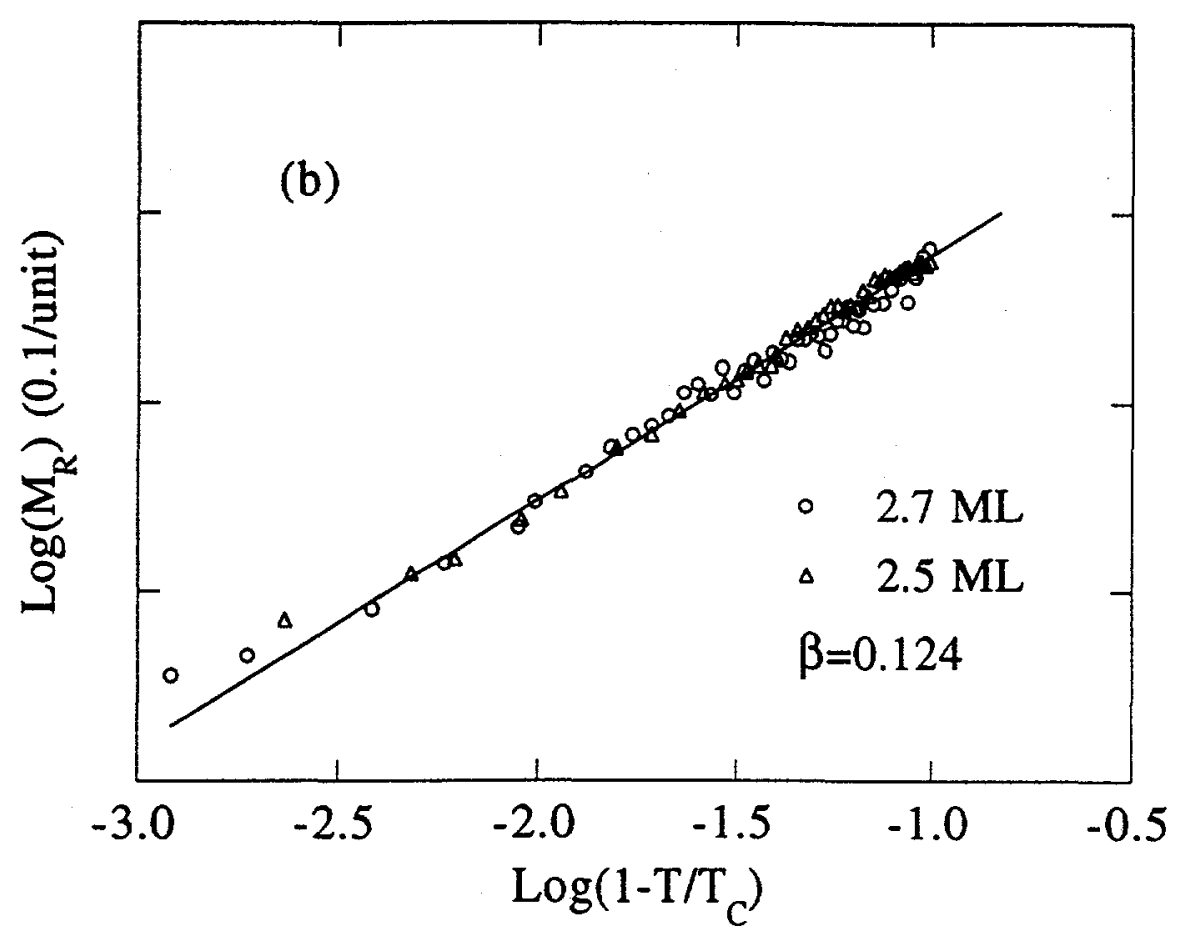




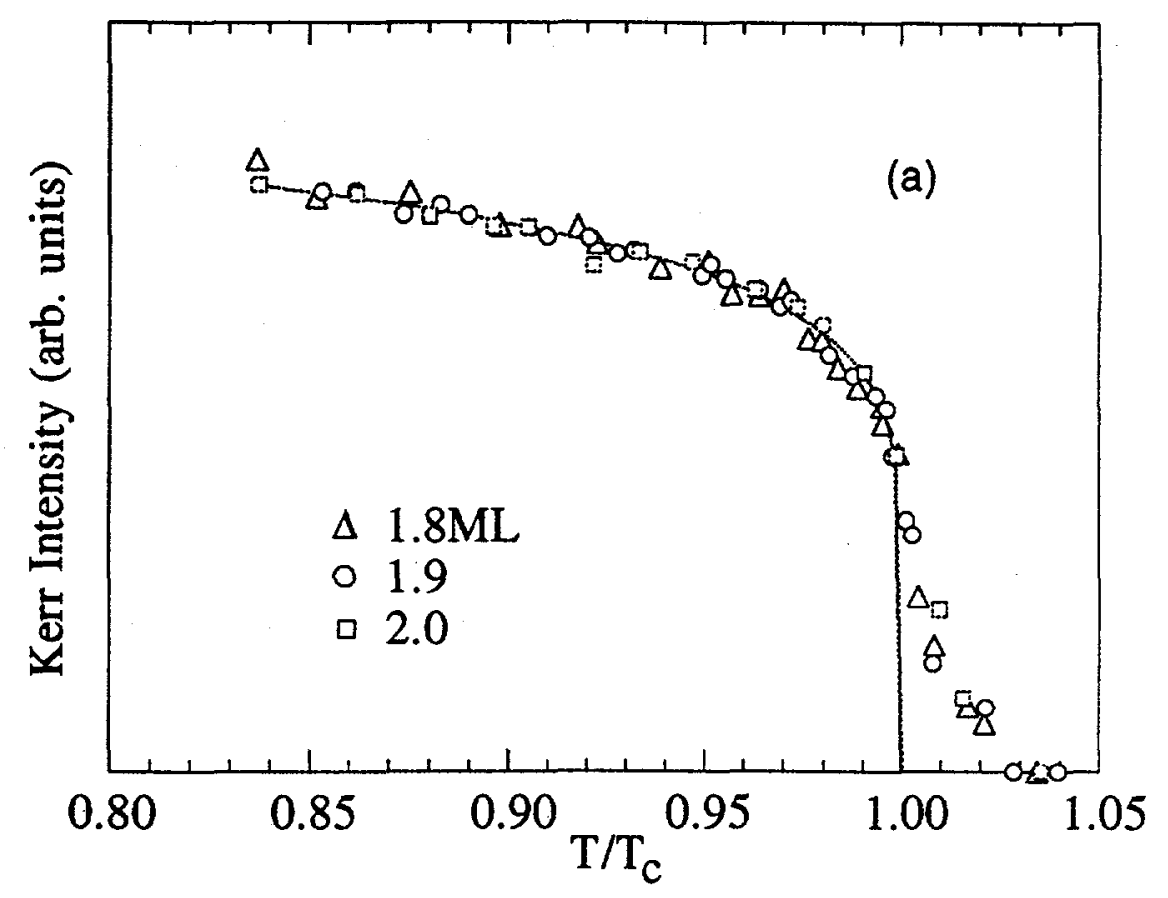




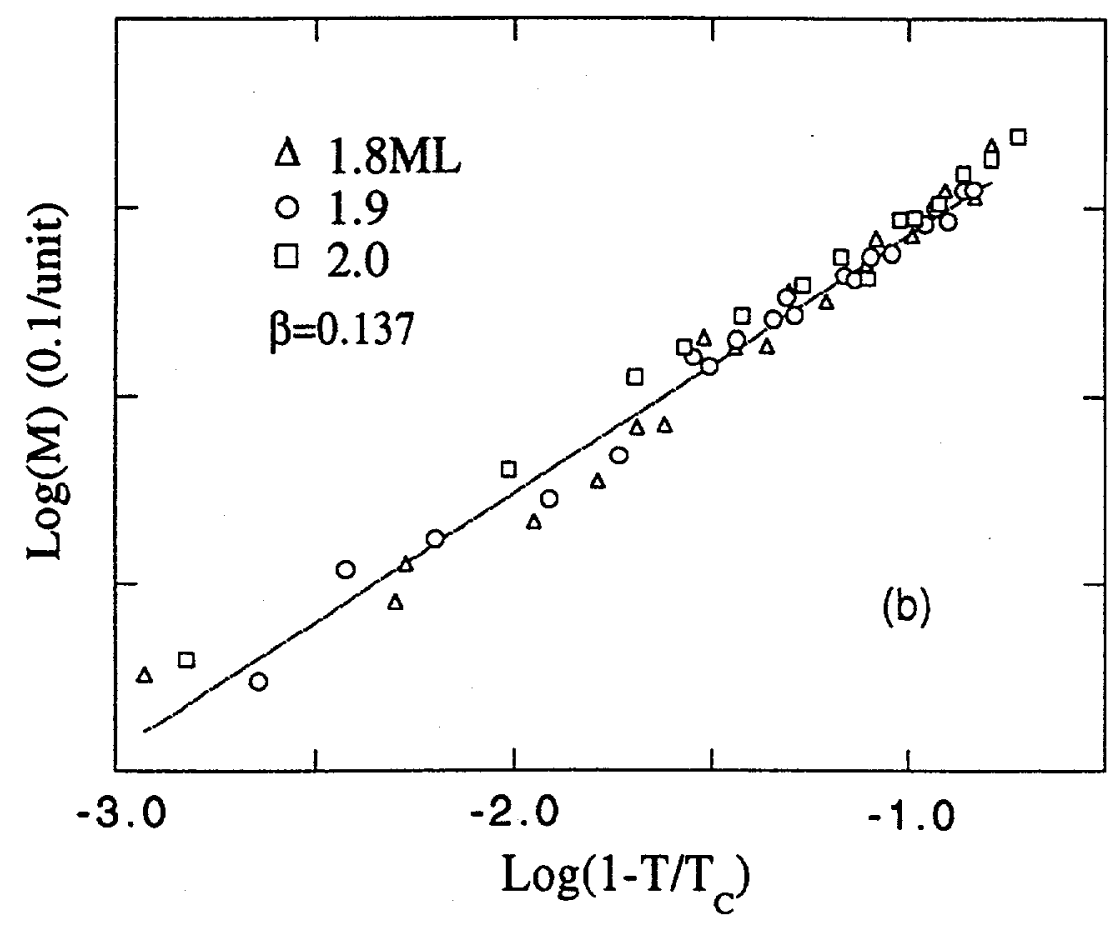




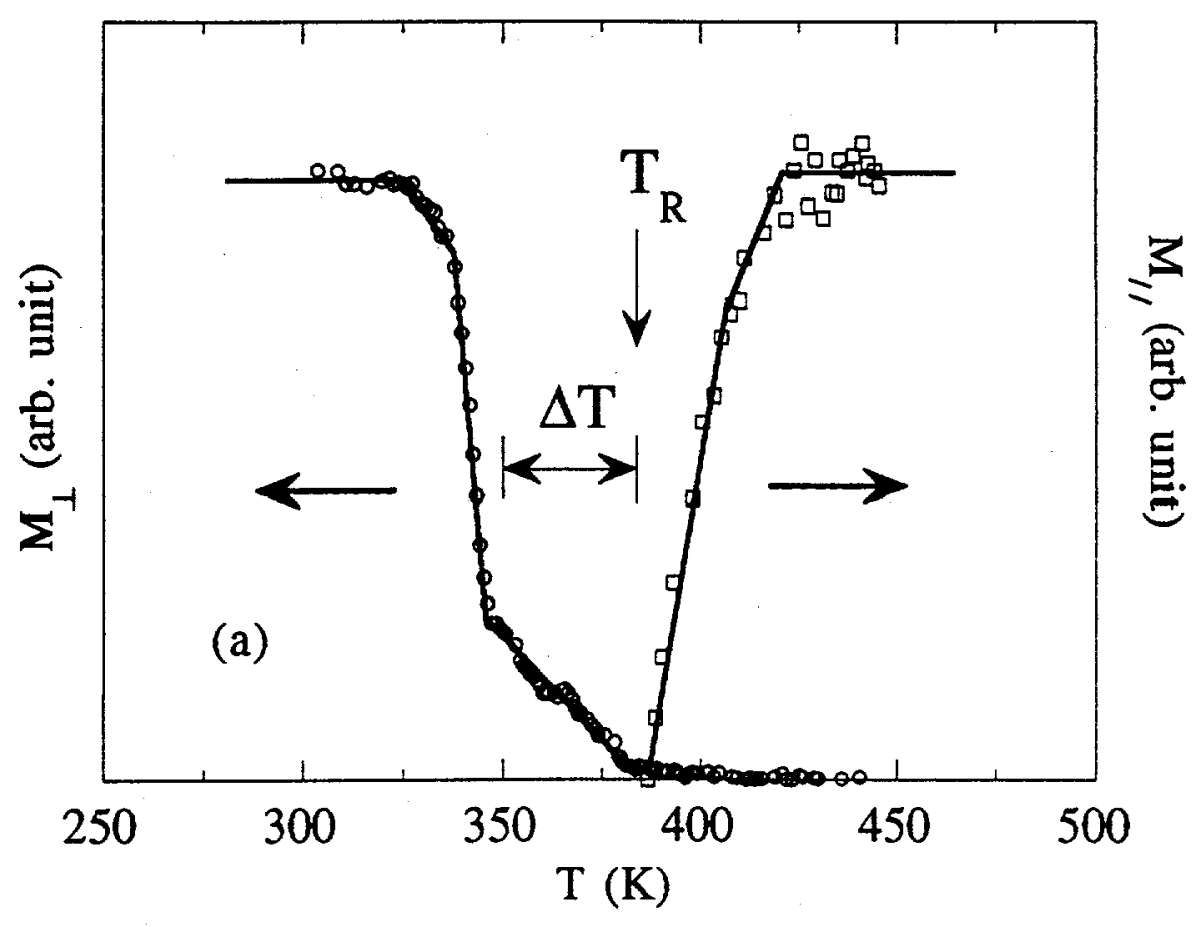




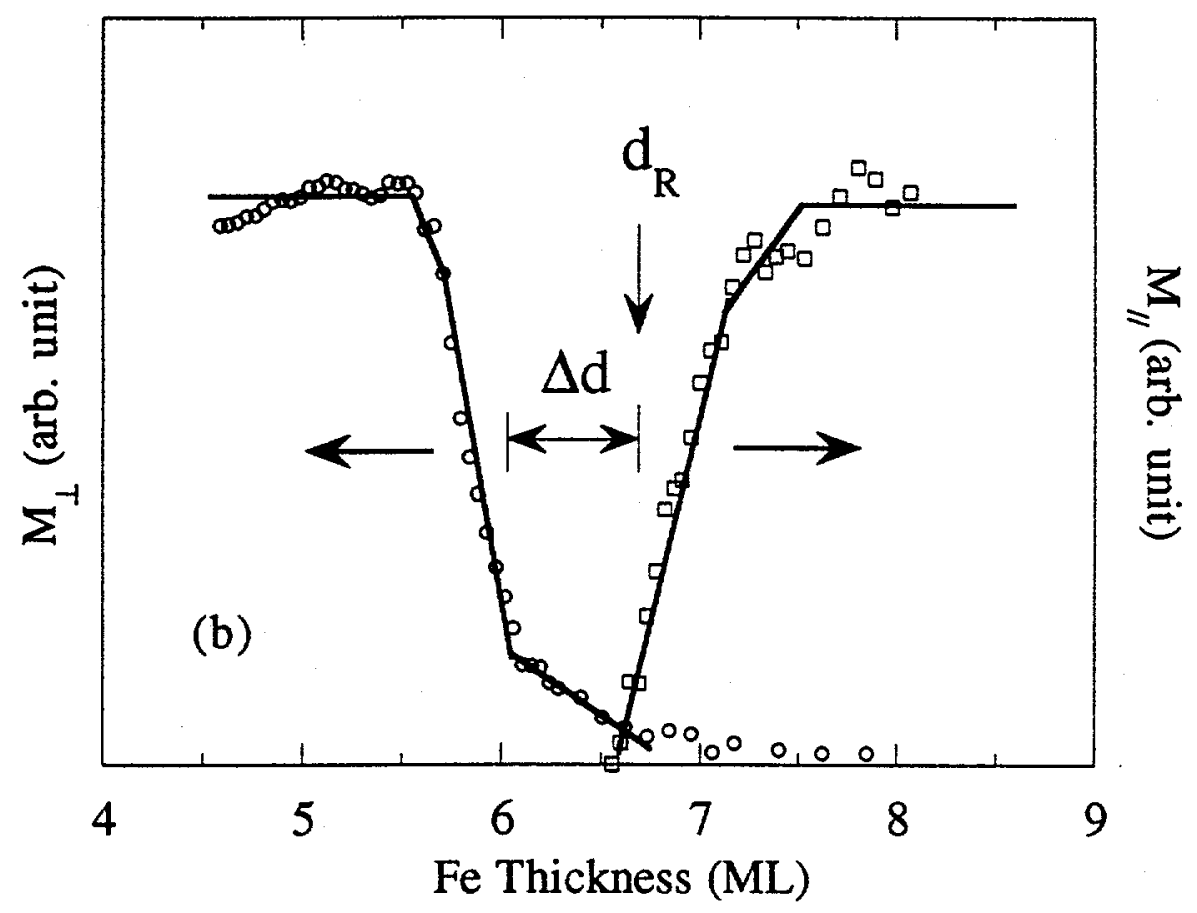




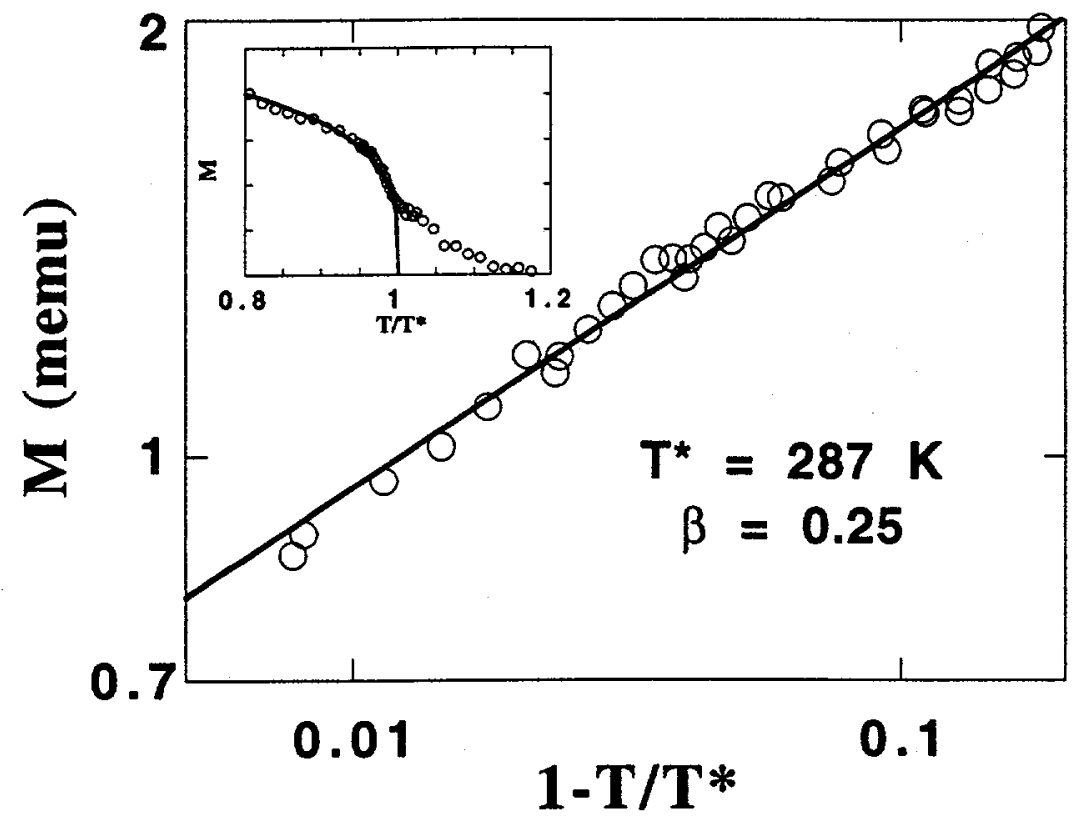




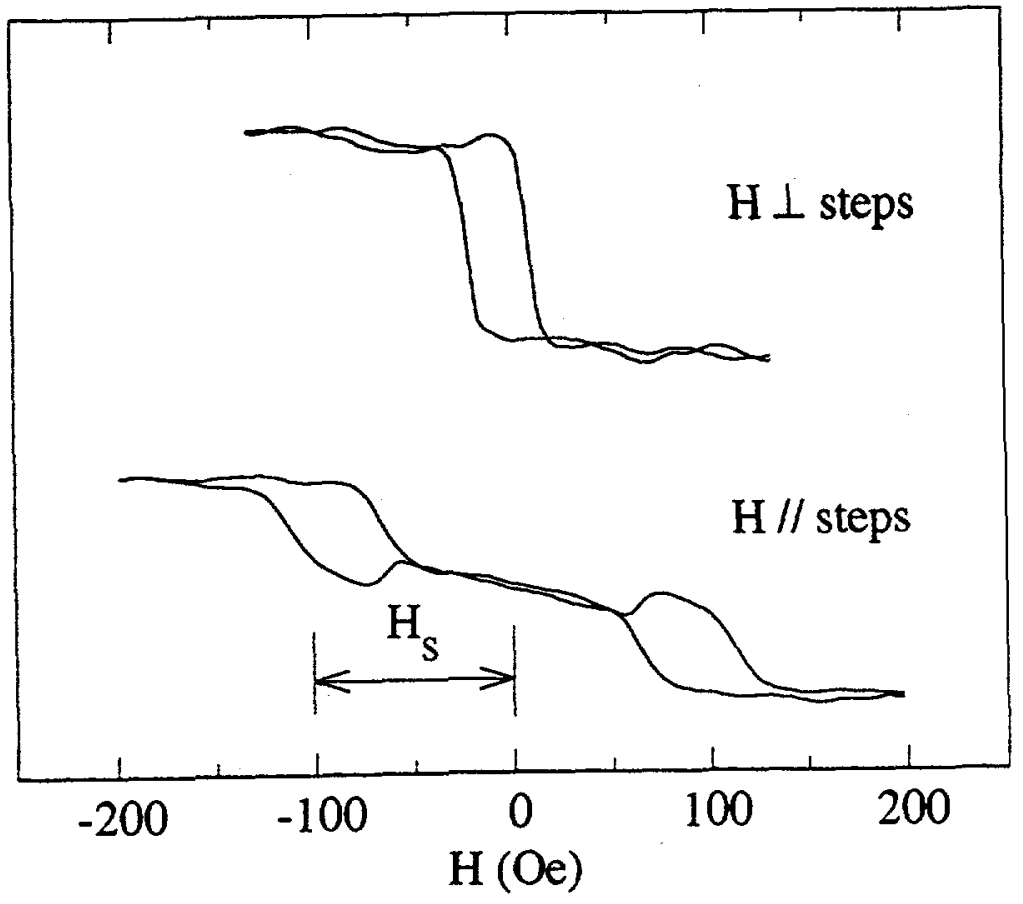




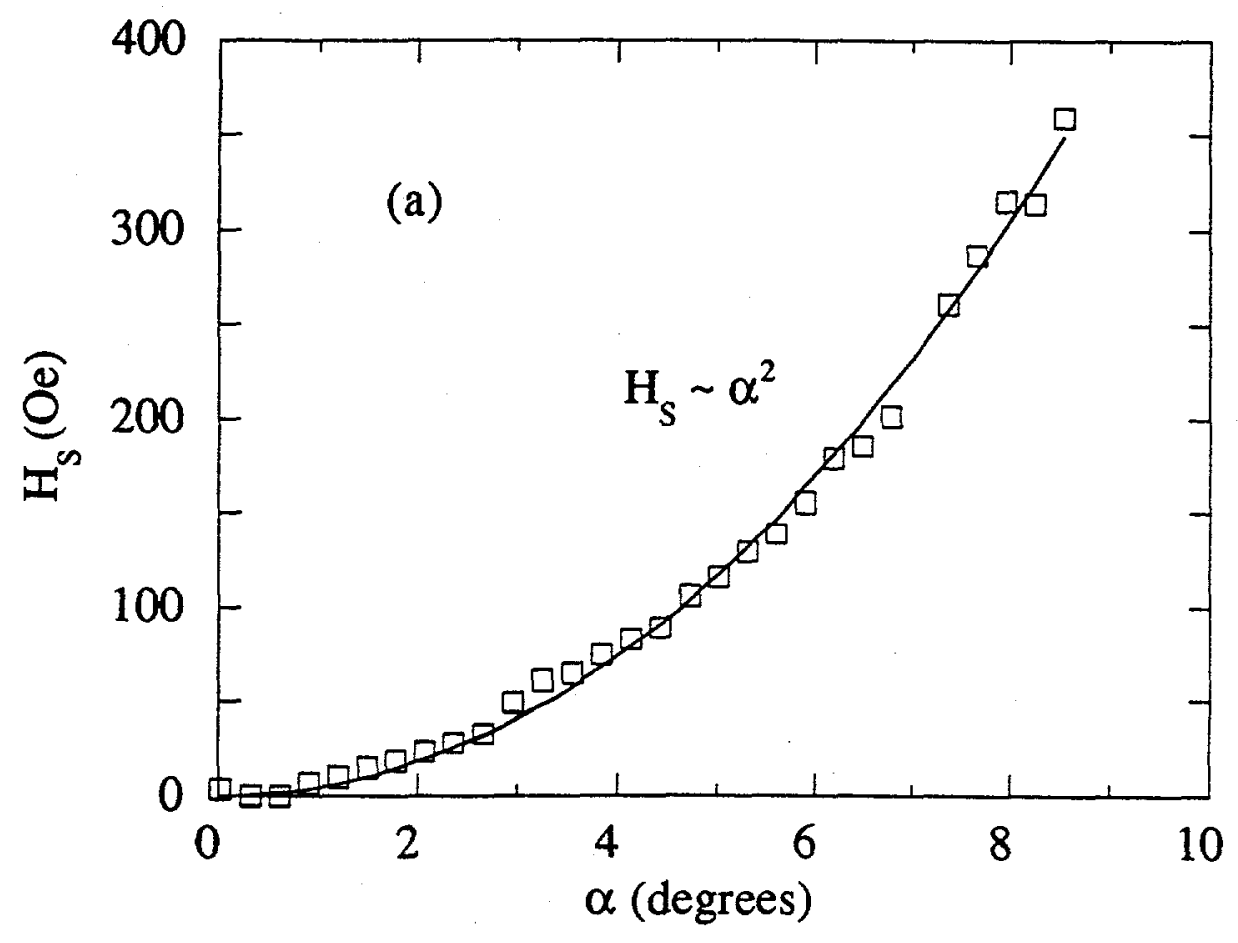




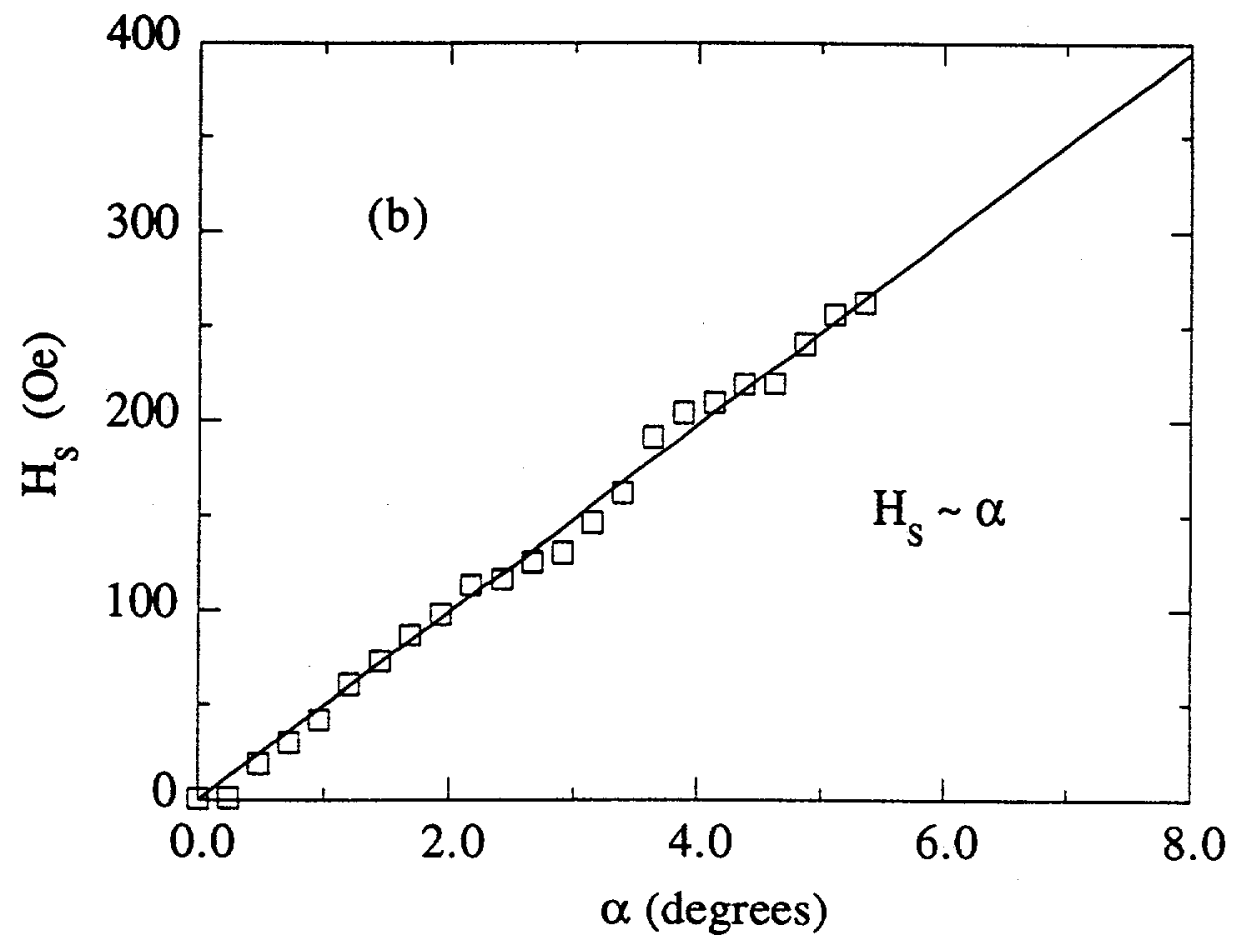




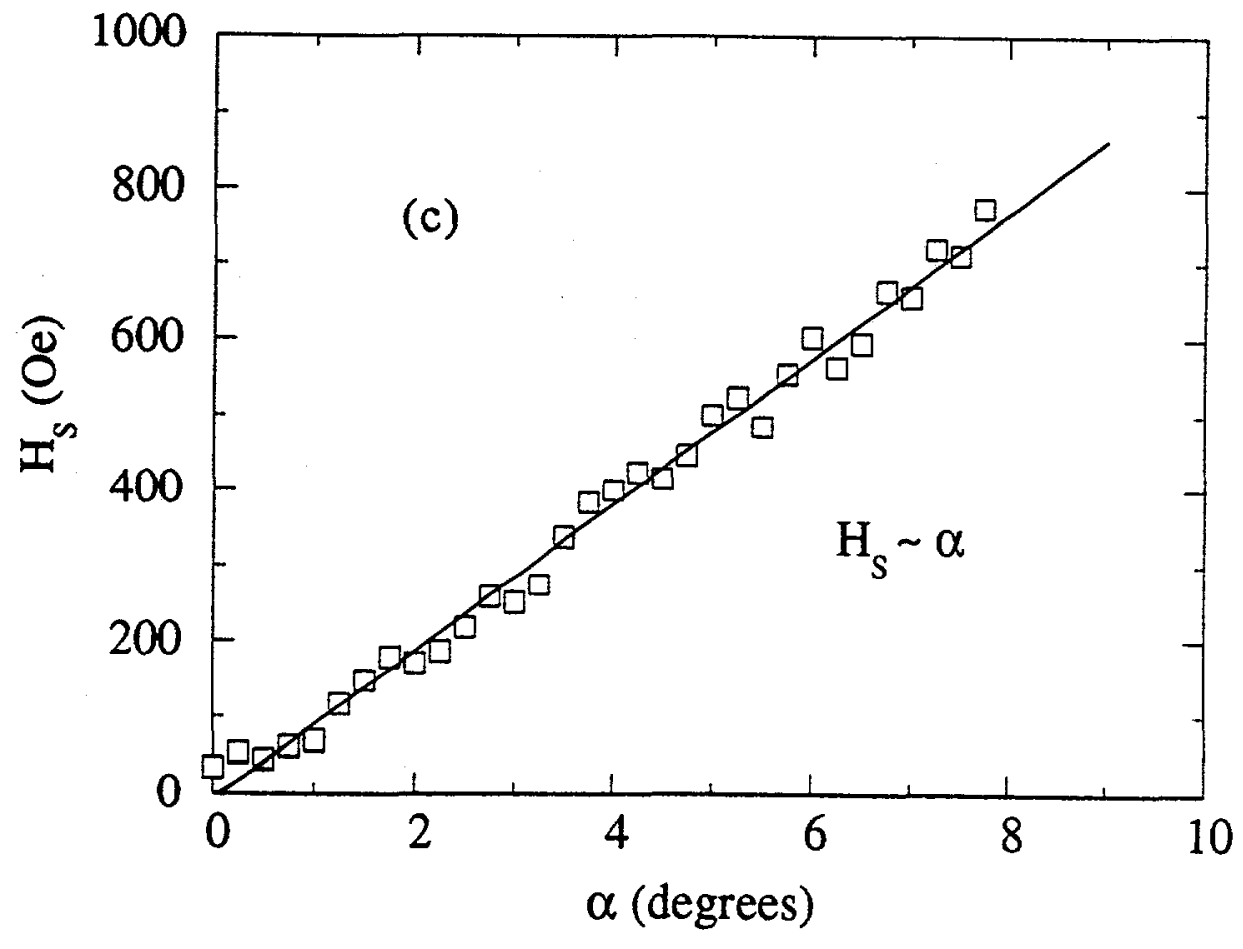

